\title{
Review of Multiferroicity in a Few Promising Nanoscale Systems
}

\author{
Aditi Sahoo ${ }^{1}$, Shubhankar Mishra ${ }^{1}$, Tania Chatterjee ${ }^{1}$, Sudipta Goswami ${ }^{1,2 *}$ and Dipten Bhattacharya ${ }^{1}$ \\ ${ }^{1}$ CSIR-Central Glass and Ceramic Research Institute, Kolkata-700032, India \\ ${ }^{2}$ Department of Solid State Physics, Kolkata-700032, India
}

*Corresponding author: Sudipta Goswami, CSIR-Central Glass and Ceramic Research Institute, Department of Solid State Physics, Indian Association for the Cultivation of Science, Kolkata-700032, India

Submission: 眥 June 01, 2018; Published: 眥 August 14, 2018

\section{Introduction}

A new dimension of functionality emerges when magnetization is switched by electric field and vice versa within a single phase compound. Multiferroics-compounds that harness multi-ferro (ferroelastic, ferroelectric, ferromagnetic) orders-realize such advancements and, therefore, for justifiable reasons, have been occupying the centre-stage of research in the area of condensed matter. Numerous applications are possible: four logic-state memory (electrical writing and magnetic reading), ac/dc magnetic field sensors, microwave resonators, phase shifters, recording read heads, multiferroic generators to name a few. For obvious reasons, the compounds that exhibit strong magnetoelectric multiferroic coupling at room temperature itself are the most sought-after ones. The nanoscale systems exhibiting remarkably large magnetoelectric coupling at room temperature could usher a new regime of nanospintronic devices. Here in this brief review, we offer glimpses of new results from three promising nanoscale single-phase and composite systems which possibly turn out to be the game changer in this area in near future.

\section{Nanoscale $\mathrm{BiFeO}_{3}$}

$\mathrm{BiFeO}_{3}$ is the quintessential room temperature multiferroic which has attracted, by far, the maximum interest [1]. Magnetoelectric coupling in nanoscale $\mathrm{BiFeO}_{3}$ has been extensively investigated using isolated nanosized particles as well as epitaxial thin films [2]. We explored the size and shape effect. While size effect has been investigated by using spherical particles, shape effect demands synthesis of particles of different shapes. For example, nano-cubes have been prepared via hydrothermal method using metal nitrate precursor solution at optimum $\mathrm{pH}$. The solution was autoclaved at $160^{\circ} \mathrm{C}$ for $72 \mathrm{~h}$. The final product was thoroughly washed by distilled water and ethanol and dried at $80{ }^{\circ} \mathrm{C}$ in a vacuum drier. The TEM image (Figure 1) of as-prepared sample shows sheet-like structure consisting of nano-cubes of $\mathrm{BiFeO}_{3}$.
Here, the individual nano-cube has an average length of $75-150 \mathrm{~nm}$ with thickness $15-25 \mathrm{~nm}$. High resolution transmission electron microscopy, shown in inset of Figure 1, depicts that the [012] plane are mainly oriented perpendicular to electron beam direction. Room temperature magnetic hysteresis loop in the inset of Figure 2 shows a small ferromagnetic ordering. Multiferroic coupling of the nano cubes have been measured via measurement of remanent polarization hysteresis loop under zero and 0.5T magnetic field (Figure 2). For this purpose, the particles were deposited on an insulating $\mathrm{Si} / \mathrm{SiO}_{2}$ substrate and $\mathrm{Ag}$ electrodes were deposited in two-probe configuration. It appears that a substantial change in the polarization occurs under the magnetic field confirming the presence of multiferroic coupling $(\sim 35 \%)$ in these $\mathrm{BiFeO}_{3}$ nano-cubes. The enhanced multiferroic coupling in $\mathrm{BiFeO}_{3}$ nanocubes compared to that in spherical particles of same size may be attributed to the influence of the shape anisotropy and thus open up new vistas of shape dependency or even quantum multiferroicity in proper one-dimensional systems.

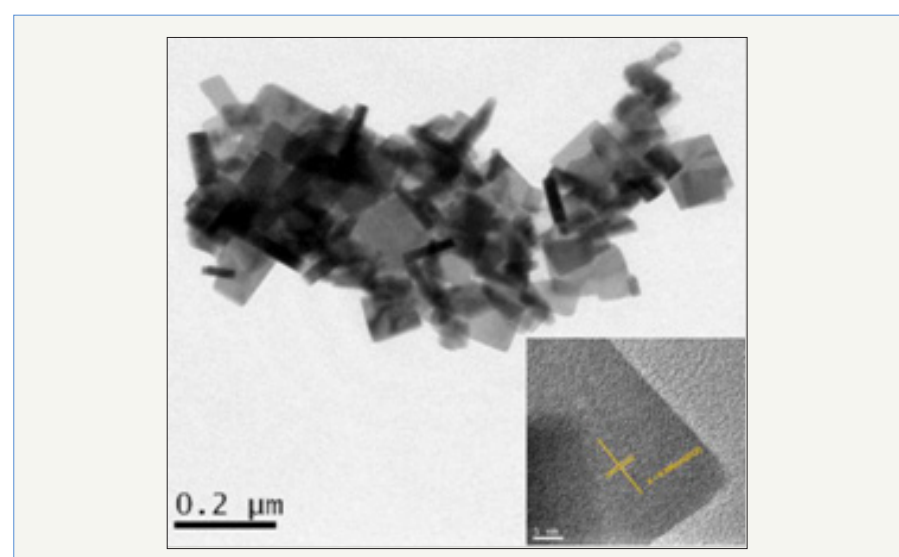

Figure 1: TEM image of $\mathrm{BiFeO}_{3}$ nano-cubes; inset: HRTEM image of the nanoparticles. 


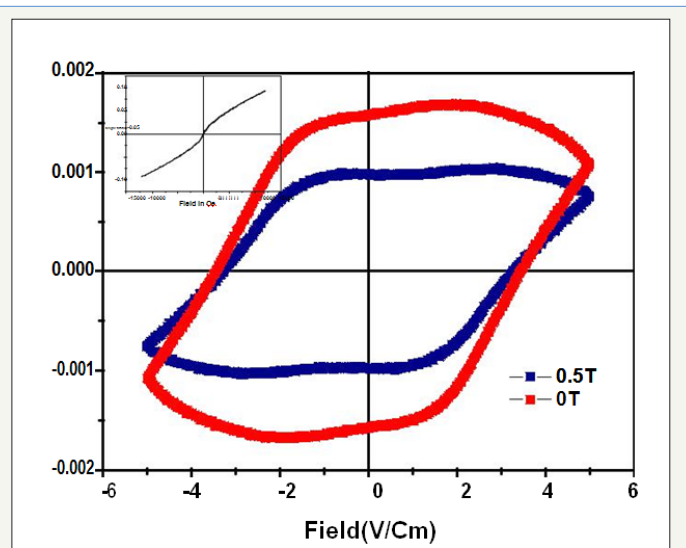

Figure 2: Multiferroic coupling in $\mathrm{BiFeO}_{3}$ nano-cubes; inset: Magnetic $\mathrm{M}-\mathrm{H}$ loop at room temperature.

\section{$\mathrm{BiFeO}_{3}$-Graphene Composite}

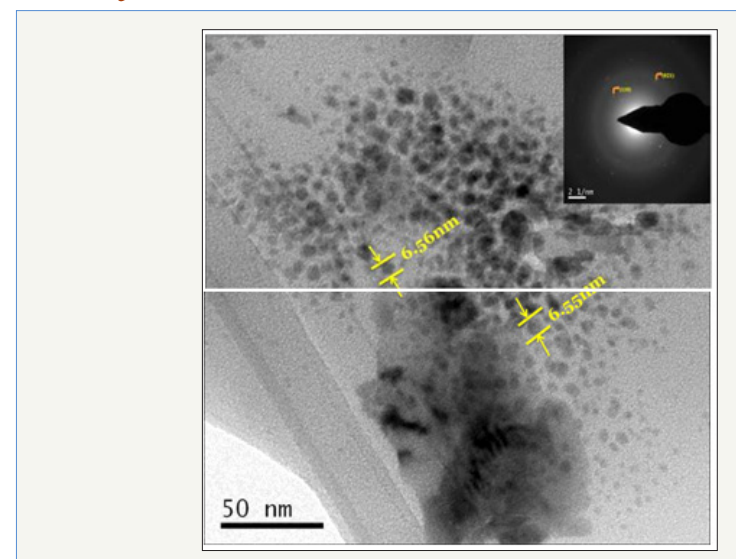

Figure 3: TEM image of Graphene- $\mathrm{BiFeO}_{3}$ nano-composite; inset: SEAD composite pattern of the nanocomposite.

Further enhancement of multiferroicity, e.g., enhancement in ferroelectric polarization, magnetic moment, and magnetoelectric coupling has been projected in the multiferroic-graphene composite systems. Recently, in a first principal calculation [3] it has been shown that there is a strong interaction between the graphene $\pi$ electrons and the magnetic $\mathrm{Mn} d$ states of a magnetoelectric multiferroic $\mathrm{BaMnO}_{3}$ within $\mathrm{BaMnO}_{3}$-graphene composite which induces a significant spin polarization in graphene. It reveals a tremendous application potential of hybrid organic-multiferroic materials in spintronics devices and many other exotic phenomena in near future. Keeping in mind this possibility, a reduced graphene oxide and $\mathrm{BiFeO}_{3}(\mathrm{rGO} / \mathrm{BFO})$ nano-composite has been prepared with an expectation of covalent bond formation between $\pi$ congugate carbon network with either $\mathrm{Bi}$ or $\mathrm{Fe}$ ion (of $\mathrm{BiFeO}_{3}$ ) via any $\mathrm{C}$ or $\mathrm{O}$ groups. This, in turn, can enhance the magnetic properties. Initially, Graphene Oxide and $\mathrm{BiFeO}_{3}$ sol were prepared separately by Modified Hummer's method and conventional sol gel method respectively. These two precursors were then mixed ( $2 \mathrm{wt} \% \mathrm{GO}$ ) and autoclaved for $24 \mathrm{~h}$ at $140{ }^{\circ} \mathrm{C}$ in a basic medium. The precipitate, thus obtained, was washed thoroughly and dried at $60{ }^{\circ} \mathrm{C}$ in vacuum. The laboratory X-Ray of the composite shows the presence of crystalline $\mathrm{BiFeO}_{3}$ phase mainly. Actually the $\mathrm{BiFeO}_{3}$ particles are embedded upon graphene oxide micro disrupted sheets with an average size of $6-10 \mathrm{~nm}$. This is also confirmed by the TEM image (Figure 3) which shows the presence of (110), (021) $\mathrm{BiFeO}_{3}$ plane in SAED pattern. Room temperature magnetic hysteresis loop (Figure 4) of the composite shows ferromagnetic behaviour and sizable enhancement in saturation magnetization.

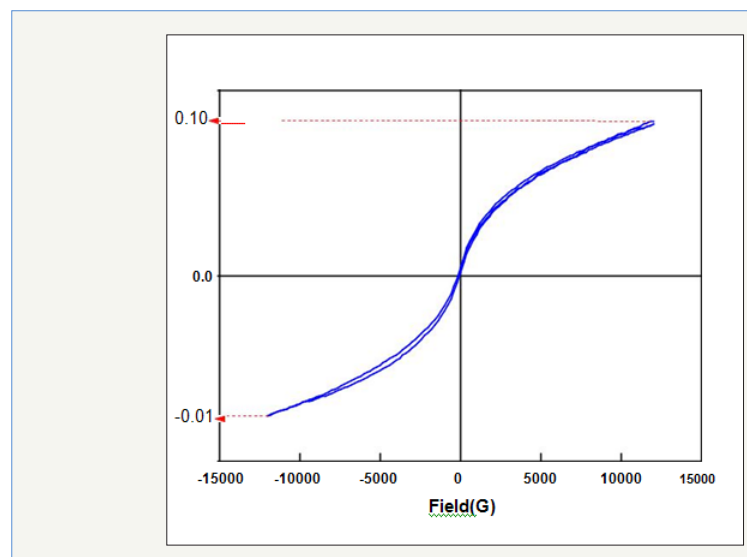

Figure 4: The room temperature $\mathrm{M}-\mathrm{H}$ loop of Graphene $\mathrm{BiFeO} 3$ nanocomposite

\section{Nanoscale Double Perovskite System}

Double perovskites materials have also drawn an extensive attention since last decades due to its multiferroic behavior and magnetoelectric coupling. $\mathrm{Y}_{2} \mathrm{NiMnO}_{6}$ being one of those double perovskite systems which possess giant dielectric tunability $[4,5]$. The dependence of the tunability on magnetic structure makes it a suitable candidate for a Type-II multiferroic. Single phase $\mathrm{Y}_{2} \mathrm{NiMnO}_{6}$ nanorods were prepared by hydrothermal method using $\mathrm{Y}\left(\mathrm{NO}_{3}\right)_{3} \cdot 6 \mathrm{H}_{2} \mathrm{O}, \mathrm{Ni}\left(\mathrm{NO}_{3}\right)_{3} \cdot 6 \mathrm{H}_{2} \mathrm{O}$ and $\mathrm{C}_{4} \mathrm{H}_{6} \mathrm{MnO}_{4} \cdot 4 \mathrm{H}_{2} \mathrm{O}$ in an autoclave at $180{ }^{\circ} \mathrm{C}$ for 24 hours in a basic medium. The precipitate thus obtained washed thoroughly and dried in vacuum at $80{ }^{\circ} \mathrm{C}$ for 4 hours followed by calcined at $1000{ }^{\circ} \mathrm{C}$ for another 4 hours. Transmission Electron Microscopy image (Figure 5) of the sample shows well defined $\mathrm{Y}_{2} \mathrm{NiMnO}_{6}$ nanorod with uniform diameter around 200nm. HRTEM image corresponds to (-111) plane with lattice spacing of $0.335 \mathrm{~nm}$ has been shown in the inset Figure 5. Multiferroic coupling (Figure 6) of the sample were measured in the same manner as stated above and substantial change in the polarization $(\sim 59 \%)$ was observed indicating $\mathrm{Y}_{2} \mathrm{NiMnO}_{6}$ nanorod to be a giant magnetoelectric multiferroic at room temperature.

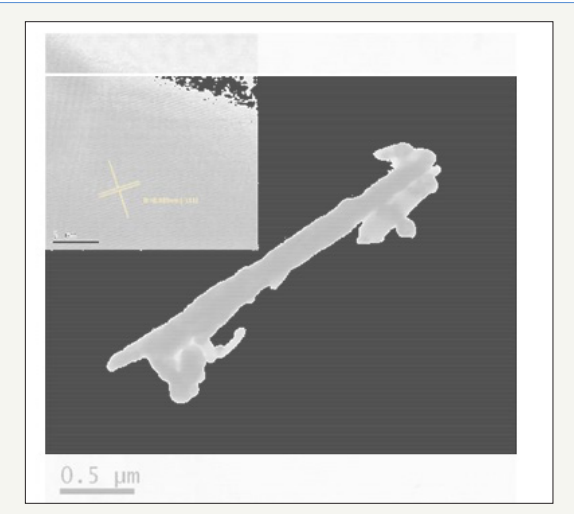

Figure 5: TEM image of Y2NiMnO6 nanotube; inset: HRTEM analysis of the nanotube. 


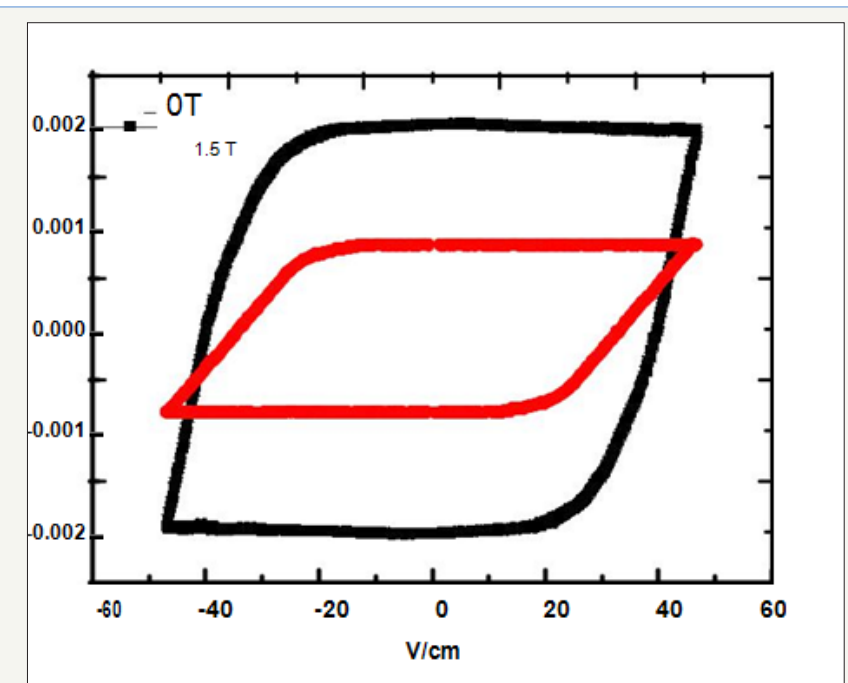

Figure 6: Multiferroic coupling in Y2NiMnO6 nanotube.

\section{Conclusion}

Even though more detailed investigation and developmental work are needed in order to utilize the remarkable enhancement in magnetoelectric multiferroic properties of these systems for realization of real-life nanospintronic devices, yet the early results from nanoscale particles of different shapes for $\mathrm{BiFeO}_{3}$, grapheme$\mathrm{BiFeO}_{3}$ composite, and nanoscale double perovskite $\mathrm{Y}_{2} \mathrm{NiMnO}_{6}$ appear to be extremely promising. In this sort review we present some highlights leaving the full details and future scope aside for a different and more comprehensive article. Nonetheless, in this brief article a taste of the fervour that is driving rigorous exploration both with conventional and newer compounds for ushering a new era of nanospintronics with multiferroics can be obtained.

\section{References}

1. Catalan G, Scott JF (2009) Physics and applications of bismuth ferrite. Adv Mat 21(4): 2463-2485.

2. Goswami S, Bhattacharya D (2016) Magnetoelectric multiferroicity at nanoscale. Sci Adv Today 2: 25227.

3. Zanolli Z (2016) Graphene-multiferroic interfaces for spintronics applications. Sci Reports 6: 31346.

4. Maiti RP, Dutta S, Mukherjee M, Mitra MK, Chakravorty D (2012) Magnetic and dielectric properties of sol-gel derived nanoparticles of double perovskite $\mathrm{Y}_{2} \mathrm{NiMnO}_{6}$. J App Phys 112: 044311.

5. Su J, Yang ZZ, Lu XM, Zhang JT, Gu L, et al. (2015) Magnetism-driven ferroelectricity in double perovskite $\mathrm{Y}_{2} \mathrm{NiMnO}_{6}$. ACS Appl Mat Interface 7(24): 13260. (c) (i) Creative Commons Attribution 4.0 International License

For possible submissions Click Here
Submit Article

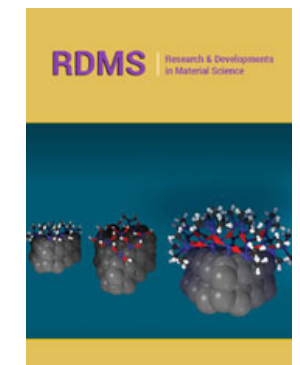

\section{Research \& Development in Material Science}

\section{Benefits of Publishing with us}

- High-level peer review and editorial services

- Freely accessible online immediately upon publication

- Authors retain the copyright to their work

- Licensing it under a Creative Commons license

- Visibility through different online platforms 